\title{
"Electron Tweezers" as a Tool for In Situ Manipulation and Processing of Individual Metal Nanoparticles in a Two-Phase Partially Molten Alloy
}

Vladimir P. Oleshko and James M. Howe

University of Virginia, Department of Materials Science \& Engineering, Charlottesville, VA 22904

Understanding physical principles for controlling the dynamic behavior of nanoparticles in inhomogeneous gas (liquid) - solid systems is becoming increasingly important for nanoscience and technology. Nondestructive trapping of micron- and nano-sized dielectric and metal particles in a liquid using a laser beam, which is refracted by the particle and transfers momentum to it, is known as a single-beam gradient force optical trap, or optical tweezers [1]. Recent experiments utilizing partially molten submicron-sized Al-Si alloy spheres and a focused electron beams in a mediumvoltage TEM [2] and theoretical calculations of linear and angular momentum transfer from an electron beam to small particles [3] indicate that optical trapping of particles using electron beams may occur. Such thermally assisted "electron tweezers" potentially can be utilized for manipulation and processing of individual nanoobjects and fabrication of assembled nanodevices with up to atomic level sensitivity and lateral resolution provided by modern electron optical systems. In this work, we demonstrate electron-beam trapping of solid nanoparticles inside opaque submicron-sized Al-Si alloy spheres using energy filtering TEM (EFTEM) and parallel electron energy-loss spectroscopy (PEELS). Thermally assisted motion of crystalline Al nanospheres confined within the partially molten Al-Si particles was initiated by the electron beam, which was used to control and to observe the trapping in real-time by plasmon spectroscopic imaging (Fig. 1). Such approaches enable in situ study of both melting and solidification in individual alloy nanoparticles and related changes of their internal structure, electronic and optical properties.

20-400 nm diameter particles of atomized Al-11.6 at. \% Si alloy, which has a eutectic (initial melting) temperature of $577^{\circ} \mathrm{C}$ and a liquidus (final melting) temperature of $581^{\circ} \mathrm{C}$ [4], were examined in a JEOL 2010F Schottky field-emission analytical electron microscope. The instrument was equipped with a Gatan Model 678 Imaging Filter operated at $197 \mathrm{kV}$. Video recording of EFTEM images was carried out using a VCR with a time resolution of 1/30 s. Each frame was then imported into a Macintosh G4 computer with a video capture board and Adobe Premier 5.0 software. PEEL spectra were acquired with an energy resolution of $1.0 \mathrm{eV}$, based on the measured FWHM of the zero-loss peak. The particles were heated in the microscope using a Gatan double-tilt $\left( \pm 30^{\circ}\right)$ heating holder with a maximum obtainable temperature of $800^{\circ} \mathrm{C}$. Real-time plasmon imaging of melting/solidification of Al nanospheres inside the submicron-sized Al-Si alloy particles was performed at $658^{\circ} \mathrm{C}$ with a $6 \mathrm{eV}$ energy window centered at the first plasmon at $15 \mathrm{eV}$.

At $620-660^{\circ} \mathrm{C}$, the alloy particles are partially molten and form a stable solid-liquid two-phase mixture. Contrast tuning using energy filtering at 15-25 energy losses was employed to visualize structural features inside the partially molten particles [5]. Real-time observations show that Al-rich solid nanospheres can be generated, steered and melted inside the submicron-sized molten binary alloy particles using temperature control and focused electron beam (Fig. 1). Thickness fringes indicate that the $40 \mathrm{~nm}$ diameter nanosphere inside the heated alloy particle is still crystalline (Fig. 1a). The crystalline Al nanosphere was observed to follow a focused electron beam as it was moved around on the particle and it also followed the beam when the stage was moved under the beam 
(Figs. 1b and 1c). Solid nanospheres inside the neighboring molten alloy particles could also be generated, manipulated and melted. Enhanced vibrations of the solid-liquid interface near the critical threshold before melting were observed at 1/30 s time resolution. The Kramers-Kronig derived complex refractive index, $\mathrm{N}=\varepsilon^{1 / 2}=\mathrm{n}+\mathrm{ik}$ (Fig. 2a), shows refraction, $\mathrm{n}=\sqrt{0.5\left(\sqrt{\varepsilon_{1}^{2}+\varepsilon_{2}^{2}}+\varepsilon_{1}\right)}$, and extinction, $\mathrm{k}=\sqrt{0.5\left(\sqrt{\varepsilon_{1}^{2}+\varepsilon_{2}^{2}}-\varepsilon_{1}\right)}$, in the range of $0-40 \mathrm{eV}$ energy loss for the solid particle and for the liquid particle, respectively. According to $[1,2]$, a frequency-dependent relative refraction, $\mathrm{n}_{\mathrm{s} / \mathrm{l}}=\mathrm{n}_{\mathrm{s}} / \mathrm{n}_{1}$ (Fig. 2b), contributes to a backward gradient force in single-beam gradient force traps, which should overcome scattering and absorption forces. As a result, the solid Al nanosphere acts as a weak positive lens, allowing it to be manipulated by an electron beam [6].

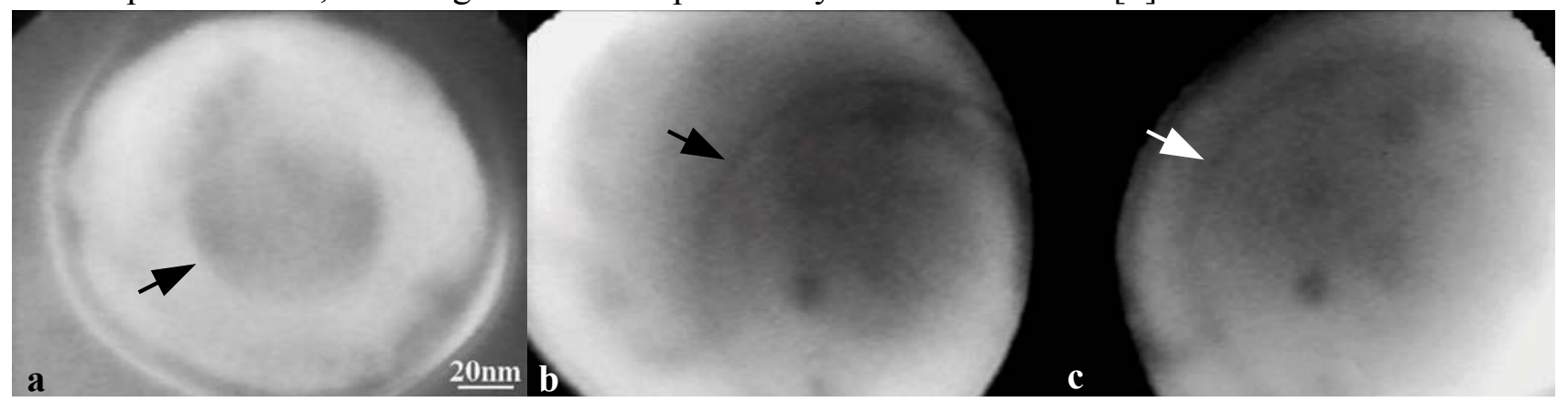

Fig. 1. Real-time in situ EFTEM video frames at $15 \mathrm{eV}$ energy loss with a $6 \mathrm{eV}$ window, $\mathrm{T}=658^{\circ} \mathrm{C}$. (a) Electron beam-assisted generation of a $40 \mathrm{~nm}$ diameter solid sphere (arrow) inside a partially molten Al-11.6 at. \% Si particle surrounded by a $\sim 10 \mathrm{~nm}$ thick oxide shell. (b, c) Steering of a second solid particle (arrows) by moving the beam and/or stage. Frame (b) is separated from frame (c) by $17 \mathrm{~s}$.
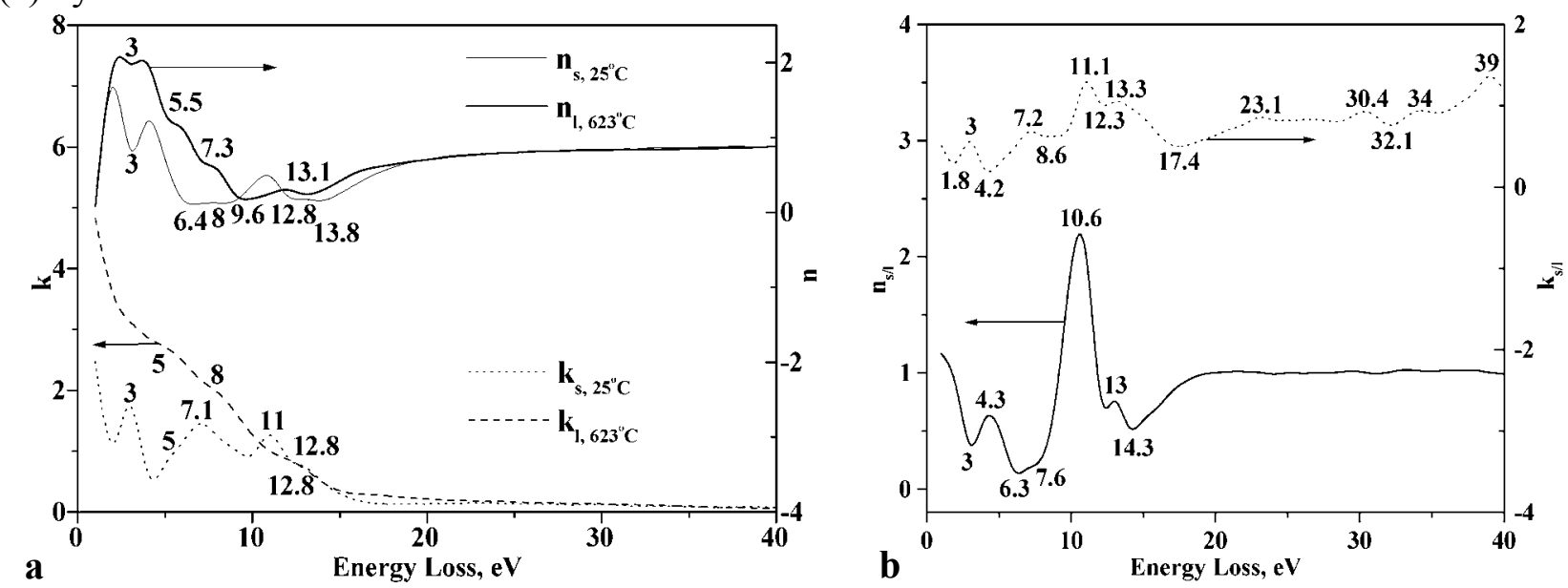

Fig. 2. (a) Complex refractive index, $\mathrm{N}=\varepsilon^{1 / 2}=\mathrm{n}+\mathrm{ik}$, and (b) relative refraction, $\mathrm{n}_{\mathrm{s} / 1}=\mathrm{n}_{\mathrm{s}, 25} / \mathrm{n}_{1,623}$, and extinction, $\mathrm{k}_{\mathrm{s} / 1}=\mathrm{k}_{\mathrm{s}, 25} / \mathrm{k}_{1,623}$, coefficients for solid $\left(\mathrm{T}=+25^{\circ} \mathrm{C}\right)$ and melted $\left(\mathrm{T}=+623^{\circ} \mathrm{C}\right)$ Al-Si particles, respectively, derived via Kramers-Kronig transformations [5].

References: [1] A. Ashkin et al., Opt. Lett. 19 (1986) 288. [2] J. M. Howe et al., J. Electron Microsc. 53 (2004) 107. [3] F. J. G. Abajo, Phys. Rev. B 70 (2004) 115422. [4] J. L. Murray, A. J. McAlister, Bull. Alloy Phase Diagrams 5 (1984) 74. [5] V. P. Oleshko, J. M. Howe, Microsc. Microanal. 10 (Suppl. 2) (2004) 350. [6] This research was supported by the US Department of Energy (Grant No. DE-FG02-01ER45918). 